\section{Investigar para visibilizar el cuidado.}

ISSN-PRINT

1794-9831

E-ISSN 2322-7028

Vol. 15 No. 1

Ene - Jun 2018

Cúcuta, Colombia

El cuidado actualmente es reconocido como algo esencial para la vida, que dio origen a múltiples profesiones con objetivos y formas diferentes de cuidar, razón por la cual no es exclusivo de enfermería. Ha sido objeto de estudio y reflexión filosófica, identificando en su universo categorías del ámbito ontológico, antropológico, epistemológico y pedagógico que envuelven, desde distintas miradas, todo lo que rodea y responde a su esencia: ser una persona cuidada, las obligaciones del cuidado, lo que hace la persona cuando cuida, la forma en que el cuidado es conocido, aprendido y enseñado. De hecho, el cuidado, más que un acto, es una actitud, un proceso que abarca más que un momento de atención o de ocupación: es una preocupación (1).

El cuidado, en tanto objeto de estudio, ha adquirido, a lo largo de los años, una estructura conceptual que ha alcanzado valor y significado en el entorno social, a pesar de las dificultades derivadas de no ser considerado un valor que sirve a intereses económicos, políticos y sociales. Dentro de este proceso, un elemento relevante lo constituye la identificación del cuidado como esencia y objetivo central de enfermería. El Consejo Internacional de Enfermería (CIE) pone de manifiesto que "la enfermería abarca los cuidados, autónomos y en colaboración, que se prestan a las personas de todas las edades, familias, grupos y comunidades, enfermos o sanos, en todos los contextos, e incluye la promoción de la salud, la prevención de la enfermedad y los cuidados de los enfermos, discapacitados y personas moribundas. Funciones esenciales de la enfermería son la defensa, el fomento de un entorno seguro, la investigación, la participación en la política de salud y en la gestión de los pacientes, los sistemas de salud y la formación" (2).

El cuidado como actividad profesional está integrado por componentes científicos, técnicos y humanos que implican acciones con sentido y orientadas al logro de resultados en la búsqueda del bienestar de las personas cuidadas; por tanto, representa una acción consciente, una actitud y un compromiso.

Los resultados del cuidado pueden ser diversos -al igual que las formas de cuidar-, concretos o abstractos, totales o parciales. La curación de una lesión es concreta, pero conseguir una sonrisa, un desahogo, el llanto o ver lo que no está expuesto del ser que es cuidado, es una forma de cuidar, es un resultado. Cuando se piensa en un cuidado objetivo, como la realización de procedimientos o una atención de emergencia, generalmente se obtiene un resultado inmediato. Sin embargo, cuando se trata de cuidado subjetivo -como el apoyo emocional o el tratamiento de enfermedades crónicas-, el resultado puede alcanzarse a largo plazo. Entonces, se puede cuidar sin resolver, puesto que el cuidado no está directamente relacionado con resultados inmediatos; los resultados también pueden ser de medio y largo plazo (3).

El concepto del cuidado como esencia de enfermería se construye desde la cotidianidad de su práctica, evoluciona y cambia paralelamente con el desarrollo del conocimiento científico y técnico, de la cultura y las creencias -tanto de los profesionales como de las personas cuidadas-, influenciado por las transformaciones sociales, las instituciones y el ambiente donde acontece.

Enfermería y otras profesiones del área de la salud enfrentan los desafíos del cuidado de salud en el contexto de una práctica permeada por profundos problemas sociales, perfiles epidemiológicos en los que prevalecen problemas de salud de carácter crónico y resurgen enfermedades infecciosas, además de transformaciones de los servicios de salud determinadas por factores económicos, acompañadas de manifestaciones de insatisfacción por parte de los usuarios ante una atención despersonalizada y deshumanizante (4-7). En este proceso, la investigación se convierte en una herramienta para la búsqueda de nuevas y mejores formas de cuidar y de construcción conocimiento en el ámbito disciplinar.

Investigar significa descubrir o examinar una cosa cuidadosamente. En el mundo de la ciencia significa la indagación sistemática y diligente para validar, transformar y generar conocimiento de manera más simple, en donde se prueba y estimula la generación de teoría (8). La investigación es esencial para el desarrollo de las profesiones de la salud; a través de

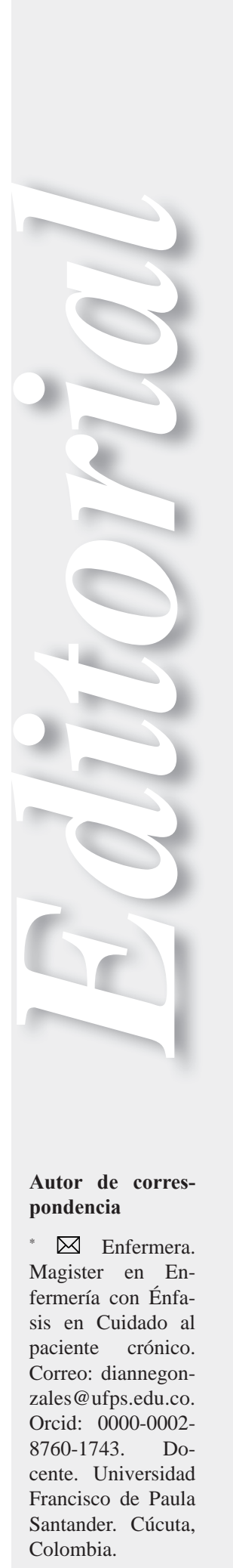


ella se genera el conocimiento científico que permite sustentar la práctica y desarrollar intervenciones para el cuidado de la salud en la promoción, prevención, atención y rehabilitación, así como el mantenimiento del bienestar.

Para una disciplina profesional como enfermería, es esencial la búsqueda de mejores formas de cuidar y de fortalecer el control de la forma como se presta la atención y se brinda cuidado. Por lo tanto, es fundamental la investigación de la atención y el cuidado para comprender como se alcanza el conocimiento que la sustenta (9).
Sin embargo, no es suficiente con que se investigue alrededor del fenómeno del cuidado: resulta indispensable que las acciones investigativas se difundan y se divulguen, puesto que la socialización del conocimiento científico es una responsabilidad de todo aquel que investiga, porque contribuye a la democratización del saber, a superar las desigualdades existentes y a debatir los resultados con la comunidad formada por especialistas en la materia (10). De esta manera, se hace visible el conocimiento que se construye y se renueva.

\section{Referencias Bibliográficas}

1. Arenas NM. El cuidado como manifestación de la condición humana. Salus [Internet] 2006. [Consultado el 12 de Noviembre de 2017]; 10(1):12-17. Disponible en: http://www.redalyc.org/ articulo.oa?id=375938979004

2. Consejo Internacional de Enfermería (CIE). Definición de Enfermería. [Internet] 2017 [Consultado el 20 de Diciembre de 2017] Disponible en: http://www.icn.ch/es/who-we-are/icn-definition-of-nursing/

3. Kuerten Rocha P, Lenise DoPrado M, De Gasperi P, Fabiane Sebold L, Waterkemper R, C-Bub MB. El cuidado y la enfermería. av.enferm. [Internet]. 2009 [consultado 20 Diciembre 2017]; 27(1):102-109. Disponible en: http://www.scielo.org.co/scielo.php?script=sci_arttext\&pid=S012145002009000100011\&lng=en.

4. Ávila Morales, JC. La deshumanización en medicina. Desde la formación al ejercicio profesional. Iatreia [Internet] 2017. [Consultado el 12 de Noviembre de 2017] 30(2):216-229. Disponible en: http:// www.redalyc.org/articulo.oa?id=180550477011

5. Poblete Troncoso M, Valenzuela Suazo V. Cuidado humanizado: un desafío para las enfermeras en los servicios hospitalarios. Acta Paul Enferm [Internet] 2007 [Consultado 20 Diciembre de 2017]; 20(4)499-503. Disponible en: http://www.scielo.br/pdf/ape/v20n4/18.pdf

6. Correa Zambrano ML. La humanización de la atención en los servicios de salud: un asunto de cuidado. Revista Cuidarte [Internet] 2016 [Consultado 21 Diciembre de 2017] 7(1)1227-31. Disponible en: https://www.revistacuidarte.org/index.php/cuidarte/article/view/300/527

7. Organización Mundial Salud. Organización Panamericana de la Salud. Salud en las Americas. [Internet] 2017. [Consultado 20 Diciembre de 2017]. Disponible en: http://www.paho.org/salud-enlas-americas-2017/wp-content/uploads/2017/09/Print-Version-Spanish.pdf

8. Durán Villalobos MM. Enfermeria: Desarrollo Teorico e Investigativo. Proyecto Innovar. Facultad de Enfermería. Universidad Nacional de Colombia. Bogotá 2001. P 142.

9. Durán Villalobos MM. La teoría, soporte de la ciencia y práctica de enfermería: tendencias. Avances en Enfermería [Internet.] 2012 [Consultado 21 Diciembre de 2017]; 30(1):9-12. Disponible en: https:// revistas.unal.edu.co/index.php/avenferm/article/view/35428/36121

10. Espinosa Santos V. Difusión y divulgación de la investigación científica. Idesia (Arica). [Internet] 2010 [Consultado 20 Diciembre 2017] 28(3):5-6. Disponible en: https://dx.doi.org/10.4067/S071834292010000300001 